\title{
GAUSSIAN BOUNDS FOR REDUCED HEAT KERNELS OF SUBELLIPTIC OPERATORS ON NILPOTENT LIE GROUPS
}

\author{
A. F. M. TER ELST and HUMBERTO PRADO
}

\begin{abstract}
We obtain Gaussian estimates for the kernels of the semigroups generated by a class of subelliptic operators $H$ acting on $L_{p}\left(\mathrm{R}^{k}\right)$. The class includes anharmonic oscillators and Schrödinger operators with external magnetic fields. The estimates imply an $H_{\infty}$-functional calculus for the operator $H$ on $L_{p}$ with $p \in\langle 1, \infty\rangle$ and in many cases the spectral $p$-independence. Moreover, we show for a subclass of operators satisfying a homogeneity property that the Riesz transforms of all orders are bounded.
\end{abstract}

\section{Introduction}

In this paper we consider a class of subelliptic operators given by a composition of differential and multiplication operators acting on $L_{p}\left(\mathrm{R}^{k}\right)$. These operators generate holomorphic semigroups which are consistent on $L_{p}\left(\mathrm{R}^{k}\right)$ for $p \in$ $[1, \infty]$. Moreover, the semigroup operators turn out to be integral operators with a smooth kernel on $\mathrm{R}^{k} \times \mathrm{R}^{k}$. Examples of such operators include the (an)harmonic oscillator and the Hamiltonian for curved magnetic fields. All these operators are naturally associated to subelliptic operators on a nilpotent Lie group.

If $H$ is a subelliptic operator affiliated to a continuous representation $U$ of a Lie group $G$ then the closure generates a holomorphic semigroup $S$ which has a representation independent kernel $K$ such that

$$
S_{t}=\int_{G} d g K_{t}(g) U(g) .
$$

For the kernel $K$ one has Gaussian bounds (see [9]). Henceforth we consider a class of representations of a nilpotent Lie group on $L_{p}\left(\mathrm{R}^{k}\right)$. Under suitable conditions we show that the semigroup $S$ has a reduced heat kernel $\kappa$ such that

$$
\left(S_{t} \varphi\right)(x)=\int_{\mathrm{R}^{k}} d y \kappa_{t}(x ; y) \varphi(y)
$$

Received April 16, 1999. 
for all $t>0, \varphi \in C_{c}^{\infty}\left(\mathrm{R}^{k}\right)$ and $x \in \mathrm{R}^{k}$. The aim of this work is to prove Gaussian bounds for $\kappa$. Previously, Gaussian bounds for reduced heat kernels have been deduced in [8] and [11] for semigroups generated by strongly elliptic operators affiliated to irreducible unitary representations of nilpotent Lie groups and in [11] for strongly elliptic operators on homogeneous spaces $G / M$ with $G$ unimodular and $M$ compact. In [18] Sikora proved off- and on-diagonal bounds for the kernels of semigroups generated by (second-order) Schrödinger operators with magnetic field and a potential of polynomial growth, satisfying a Nash inequality. The novelty of this paper is that the operators are weighted subcoercive instead of strongly elliptic. As a consequence of the Gaussian bounds for the kernel we obtain that $H$ has a bounded $H_{\infty}$-functional calculus on all the $L_{p}$-spaces with $p \in\langle 1, \infty\rangle$ and also in many cases the $p$-independence of the spectrum of $H$. We also show that the Riesz transforms are bounded on $L_{p}$ for $p \in\langle 1, \infty\rangle$.

Typical examples for the second order operators are the spinless particles of mass $m$ in an external magnetic field $\vec{B}$ where $\vec{B}$ is a polynomial. Then the Hamiltonian is given by $H=\frac{1}{2 m}\left(\vec{p}-\frac{e}{c} \vec{A}\right)^{2}$ where $\vec{p}=-i \hbar \vec{\nabla}$ and $\vec{A}$ is a polynomial vector potential satisfying $\vec{B}=\vec{\nabla} \times \vec{A}$, see [13] and [19]. Other examples are the anharmonic oscillators $\left(-\partial^{2} / \partial x^{2}\right)^{j}+x^{2 n}$, with $j, n \in \mathrm{N}$.

Throughout the following let $G$ be a connected nilpotent Lie group with Lie algebra $\mathrm{g}$. Then the exponential mapping on $\mathrm{g}$ is surjective. Let $a_{1}, \ldots, a_{d^{\prime}}$ be an algebraic basis of g, i.e., $a_{1}, \ldots, a_{d^{\prime}}$ are independent and together with their multi-commutators span g. Let $U$ be a continuous representation of $G$ in a Banach space $\mathscr{X}$. For $i \in\left\{1, \ldots, d^{\prime}\right\}$ let $A_{i}=d U\left(a_{i}\right)$ be the infinitesimal generator of the one parameter group $t \mapsto U\left(\exp \left(-t a_{i}\right)\right)$. We also need multiindex notation. Set $J\left(d^{\prime}\right)=\bigcup_{n=0}^{\infty}\left\{1, \ldots, d^{\prime}\right\}^{n}$. If $\alpha=\left(i_{1}, \ldots, i_{n}\right) \in J\left(d^{\prime}\right)$ define $A^{\alpha}=A_{i_{1}} \ldots A_{i_{n}}$. Generally we adopt the notation of [9].

The representations that we consider in this paper are of the following type. First we assume that there exist $a_{d^{\prime}+1}, \ldots, a_{d} \in \mathfrak{g}$ such that $a_{1}, \ldots, a_{d}$ is a basis for $\mathfrak{g}$ and $[\mathfrak{g}, \mathfrak{g}] \subset \operatorname{span}\left\{a_{d^{\prime}+1}, \ldots, a_{d}\right\}$. Secondly, let $k \leq d^{\prime}$. For $p \in[1, \infty]$ let $U$ be a representation of $G$ in $L_{p}\left(\mathrm{R}^{k}\right)$ of the form

$$
(U(\exp a) \varphi)(x)=e^{i E(a, x)} \varphi\left(x+\xi_{a}^{(0)}\right)
$$

for all $\varphi \in C_{c}^{\infty}\left(\mathrm{R}^{k}\right)$, where $E: \mathfrak{g} \times \mathrm{R}^{k} \rightarrow \mathrm{R}$ is a real polynomial and

$$
\xi_{a}^{(0)}=\left(\xi_{1}, \ldots, \xi_{k}\right)
$$

for all $a=\sum_{i=1}^{d} \xi_{i} a_{i} \in \mathfrak{g}$. It is straightforward to see that the representation is a continuous representation acting on $L_{p}\left(\mathrm{R}^{k}\right)$ for all $p \in[1, \infty]$. 
Fix $n_{1}, \ldots, n_{d^{\prime}} \in \mathrm{N}$ and set

$$
H=\sum_{i=1}^{d^{\prime}}(-1)^{n_{i}} A_{i}^{2 n_{i}}
$$

with domain

$$
D(H)=\bigcap_{\substack{\alpha \in J\left(d^{\prime}\right) \\\|\alpha\| \leq m}} D\left(A^{\alpha}\right)
$$

where $m=2 \operatorname{lcm}\left(n_{1}, \ldots, n_{d^{\prime}}\right), w_{i}=\left(2 n_{i}\right)^{-1} m$ for all $i \in\left\{1, \ldots, d^{\prime}\right\}$ and $\|\alpha\|=w_{i_{1}}+\ldots+w_{i_{n}}$ if $\alpha=\left(i_{1}, \ldots, i_{n}\right) \in J\left(d^{\prime}\right)$. Define the modulus $\|\cdot\|: R^{k} \rightarrow[0, \infty\rangle$ by

$$
\|x\|^{2 w}=\sum_{i=1}^{k}\left|x_{i}\right|^{2 w / w_{i}}
$$

where $x=\left(x_{1}, \ldots, x_{k}\right)$ and $w=\operatorname{lcm}\left(w_{1}, \ldots, w_{k}\right)$. The main result of this paper is the next theorem.

TheOREM 1.1. Let $p \in[1, \infty]$. Then the following are satisfied.

I. The closure $\bar{H}$ of $H$ generates a semigroup $S$ in $L_{p}\left(\mathrm{R}^{k}\right)$, which is holomorphic in the right half-plane.

II. For all $t>0$ the semigroup operator $S_{t}$ has a smooth kernel $\kappa_{t} \in$ $C^{\infty}\left(\mathrm{R}^{k} \times \mathrm{R}^{k}\right)$ such that the maps $x \mapsto \kappa_{t}\left(x ; y_{0}\right)$ and $y \mapsto \kappa_{t}\left(x_{0} ; y\right)$ belong to the Schwartz space $\mathscr{S}\left(\mathrm{R}^{k}\right)$ for all $x_{0}, y_{0} \in \mathrm{R}^{k}$ and

$$
\left(S_{t} \varphi\right)(x)=\int_{\mathrm{R}^{k}} d y \kappa_{t}(x ; y) \varphi(y)
$$

for all $\varphi \in L_{p}\left(\mathrm{R}^{k}\right)$ and (a.e.) $x \in \mathrm{R}^{k}$.

III. There exist $c, \tau>0$ such that

$$
\left|\kappa_{t}(x ; y)\right| \leq c t^{-Q / m} e^{-\tau\left(\|x-y\|^{m} t^{-1}\right)^{1 /(m-1)}}
$$

for all $t>0$ and $x, y \in \mathrm{R}^{k}$, where $Q=w_{1}+\ldots+w_{k}$. Moreover, if $A_{i}$ and $B_{i}$ denote the left derivative of $\kappa_{t}$ with respect to the first and second variable, respectively, and $A^{\alpha}$ and $B^{\beta}$ the corresponding multiderivatives, then for all $\alpha, \beta \in J\left(d^{\prime}\right)$ there exist $c, \tau>0$ such that

$$
\left|\left(A^{\alpha} B^{\beta} \kappa_{t}\right)(x ; y)\right| \leq c t^{-(Q+\|\alpha\|+\|\beta\|) / m} e^{-\tau\left(\|x-y\|^{m} t^{-1}\right)^{1 /(m-1)}}
$$

for all $t>0$ and $x, y \in \mathrm{R}^{k}$. 
IV. For all $p \in\langle 1, \infty\rangle$ the operator $H$ is closed and for all $\alpha \in J\left(d^{\prime}\right)$ one has $D\left(H^{\|\alpha\| / m}\right) \subset D\left(A^{\alpha}\right)$. Moreover, there exists a $c>0$ such that

$$
\left\|A^{\alpha} \varphi\right\|_{p} \leq c\left\|H^{\|\alpha\| / m} \varphi\right\|_{p}
$$

for all $\varphi \in D\left(H^{\|\alpha\| / m}\right)$.

Statement $\mathrm{I}$ is a direct consequence of [9]. To be precise, it follows from Example 4.4, Proposition 11.3 and Theorem 1.1 of [9]. The sketch of the proof of the other three statements is as follows. The directions $a_{1}, \ldots, a_{d^{\prime}}$ with the weights $w_{1}, \ldots, w_{d^{\prime}}$ make the operator $H$ homogeneous. Unfortunately these weights do not in general allow one to define a family of dilations on $g$. This problem, however, can be circumvented by lifting the representation to a free nilpotent group $\widetilde{G}$. Then the semigroup has a kernel $\widetilde{K}$ on $\widetilde{G}$ and one can relate the reduced heat kernel $\kappa$ with $\widetilde{K}$. The Gaussian bounds for $\kappa$ follow by a projection and a scaling argument from the Gaussian bounds for $\widetilde{K}$. Finally the boundedness of the Riesz transforms follows from transference.

Although Theorem 1.1 is formulated for operators $H$ which are sums of even powers, the conclusions of the theorem are with small modifications also valid for a larger class of operators affiliated to representations of the form (1) of the group $G$. In Section 2 we prove Statements II and III of Theorem 1.1 in the generalized theorem and in Section 3 we give applications and examples. Finally, in Section 4 we discuss the boundedness of the Riesz transforms in case the operator is homogeneous. In that section the representation can be any induced representation from a character and the representation does not have to be of the form (1). In particular the bounds are valid for any basis realization of an irreducible unitary representation.

\section{Gaussian bounds}

Before we can define the operators for which the generalization of Theorem 1.1 is valid we have to introduce a suitable free Lie group.

Let $a_{1}, \ldots, a_{d^{\prime}}$ be an algebraic basis of the Lie algebra $g$ of a connected nilpotent Lie group $G$ and let $w_{1}, \ldots, w_{d^{\prime}} \in \mathrm{N}$ be weights. For $\alpha=\left(i_{1}, \ldots, i_{n}\right) \in$ $J\left(d^{\prime}\right)$ set $\|\alpha\|=w_{i_{1}}+\ldots+w_{i_{n}}$. Let

$r=\max \left\{\|\alpha\|: \alpha=\left(i_{1}, \ldots, i_{n}\right) \in J\left(d^{\prime}\right)\right.$ and $\left.\left[a_{i_{1}},\left[\ldots\left[a_{i_{n-1}}, a_{i_{n}}\right] \ldots\right]\right] \neq 0\right\}$.

Let $\tilde{\mathfrak{g}}$ be the nilpotent Lie algebra with $d^{\prime}$ generators $\tilde{a}_{1}, \ldots, \tilde{a}_{d^{\prime}}$ which is free of weighted step $r$. So $\tilde{g}$ is the quotient of the free Lie algebra with $d^{\prime}$ generators by the ideal spanned by the commutators $\left[\tilde{a}_{i_{1}},\left[\ldots\left[\tilde{a}_{i_{n-1}}, \tilde{a}_{i_{n}}\right] \ldots\right]\right]$ with $\left\|\left(i_{1}, \ldots, i_{n}\right)\right\| \geq r+1$. We give $\tilde{a}_{i}$ the weight $w_{i}$ for all $i \in\left\{1, \ldots, d^{\prime}\right\}$. Then there exists a family $\left(\gamma_{t}\right)_{t>0}$ of dilations of $\tilde{\mathfrak{g}}$ such that $\gamma_{t}\left(\tilde{a}_{i}\right)=t^{w_{i}} \tilde{a}_{i}$ 
for all $t>0$ and $i \in\left\{1, \ldots, d^{\prime}\right\}$ (see [17] or [9], Example 2.7). Moreover, there exist $\tilde{a}_{d^{\prime}+1}, \ldots, \tilde{a}_{\tilde{d}} \in \tilde{\mathfrak{g}}$ and $w_{d^{\prime}+1}, \ldots, w_{\tilde{d}} \in \mathrm{N}$ such that $\tilde{a}_{1}, \ldots, \tilde{a}_{\tilde{d}}$ is a basis for $\tilde{\mathfrak{g}}$ and $\gamma_{t}\left(\tilde{a}_{i}\right)=t^{w_{i}} \tilde{a}_{i}$ for all $t>0$ and $i \in\left\{d^{\prime}+1, \ldots, \tilde{d}\right\}$. Then $[\tilde{\mathfrak{g}}, \tilde{\mathfrak{g}}]=\operatorname{span}\left\{\tilde{a}_{d^{\prime}+1}, \ldots, \tilde{a}_{\tilde{d}}\right\}$ (cf. [9] Example 2.6).

Let $m \in \mathrm{N}$ be such that $m \in 2 w_{i} \mathrm{~N}$ for all $i \in\left\{1, \ldots, d^{\prime}\right\}$ and for all $\alpha \in J\left(d^{\prime}\right)$ with $\|\alpha\| \leq m$ let $c_{\alpha} \in \mathbf{C}$. Moreover, let $\widetilde{A}_{i}=d L_{\widetilde{G}}\left(\tilde{a}_{i}\right)$ for all $i \in\left\{1, \ldots, d^{\prime}\right\}$, where $\widetilde{G}$ is the connected simply connected Lie group with Lie algebra $\tilde{g}$ and $L_{\widetilde{G}}$ is the left regular representation of $\widetilde{G}$ on $L_{2}(\widetilde{G})$. Set

$$
\widetilde{H}=\sum_{\|\alpha\| \leq m} c_{\alpha} \widetilde{A}^{\alpha}
$$

with domain $D(\widetilde{H})=\bigcap_{\|\alpha\| \leq m} D\left(\widetilde{A}^{\alpha}\right)$ and assume that $\widetilde{H}$ is a weighted subcoercive operator, i.e., there exist $\mu, v>0$ such that

$$
\operatorname{Re}(\varphi, \tilde{H} \varphi) \geq \mu \sum_{\|\alpha\|=m / 2}\left\|\tilde{A}^{\alpha} \varphi\right\|_{\tilde{2}}^{2}-v\|\varphi\|_{\tilde{2}}^{2}
$$

for all $\varphi \in C_{c}^{\infty}(\widetilde{G})$, that is, $\widetilde{H}$ satisfies a Gårding inequality on $\widetilde{G}$ (see [9]). Here $\|\cdot\|_{\tilde{2}}$ is the norm on $L_{2}(\widetilde{G})$.

Let $U$ be a representation of $G$ in $L_{p}\left(\mathrm{R}^{k}\right)$ of the form (1). We consider the analogue operator

$$
H=\sum_{\|\alpha\| \leq m} c_{\alpha} A^{\alpha}
$$

with domain $D(H)=\bigcap_{\|\alpha\| \leq m} D\left(A^{\alpha}\right)$ and the same coefficients as the operator $\widetilde{H}$.

THEOREM 2.1. Let $U$ be a representation of the form (1) and

$$
H=\sum_{\|\alpha\| \leq m} c_{\alpha} A^{\alpha}
$$

as above. Let $p \in[1, \infty]$. Then the following are satisfied.

I The closure $\bar{H}$ of $H$ generates a semigroup $S$ in $L_{p}\left(\mathrm{R}^{k}\right)$, which is holomorphic in a p-independent sector.

II For all $t>0$ the semigroup operator $S_{t}$ has a smooth kernel $\kappa_{t} \in$ $C^{\infty}\left(\mathrm{R}^{k} \times \mathrm{R}^{k}\right)$ such that the maps $x \mapsto \kappa_{t}\left(x ; y_{0}\right)$ and $y \mapsto \kappa_{t}\left(x_{0} ; y\right)$ belong to the Schwartz space $\mathscr{S}\left(\mathrm{R}^{k}\right)$ for all $x_{0}, y_{0} \in \mathrm{R}^{k}$ and

$$
\left(S_{t} \varphi\right)(x)=\int_{\mathrm{R}^{k}} d y \kappa_{t}(x ; y) \varphi(y)
$$


for all $\varphi \in L_{p}\left(\mathrm{R}^{k}\right)$ and (a.e.) $x \in \mathrm{R}^{k}$.

III For all $\alpha, \beta \in J\left(d^{\prime}\right)$ there exist $c, \tau>0$ and $\omega \in \mathrm{R}$ such that

$$
\left|\left(A^{\alpha} B^{\beta} \kappa_{t}\right)(x ; y)\right| \leq c t^{-(Q+\|\alpha\|+\|\beta\|) / m} e^{\omega t} e^{-\tau\left(\|x-y\|^{m} t^{-1}\right)^{1 /(m-1)}}
$$

for all $t>0$ and $x, y \in \mathrm{R}^{k}$, where $Q=w_{1}+\ldots+w_{k}$ and the modulus $\|\cdot\|$ on $\mathrm{R}^{k}$ is as in (2).

Moreover, if $H$ is a pure m-thorderoperator, i.e., $H=\sum_{\|\alpha\|=m} c_{\alpha} A^{\alpha}$, then $\omega$ can be taken equal to 0 .

IV The Schwartz space $\mathscr{S}\left(\mathrm{R}^{k}\right)$ is a core for $\bar{H}$.

Proof. Statement I follows from Proposition 11.3 and Theorem 1.1 of [9].

Next, since $\tilde{\mathfrak{g}}$ is free of weighted step $r$ there exists a unique Lie algebra homomorphism $\pi: \tilde{\mathrm{g}} \rightarrow \mathfrak{g}$ such that $\pi\left(\tilde{a}_{i}\right)=a_{i}$ for all $i \in\left\{1, \ldots, d^{\prime}\right\}$. The Lie algebra homomorphism $\pi$ lifts to a Lie group homomorphism $\Phi$ from $\widetilde{G}$ onto $G$. For $\tilde{g} \in \widetilde{G}$ define $\widetilde{U}(\tilde{g})=U(\Phi(\tilde{g}))$. Then $\widetilde{U}$ is a continuous representation of $\widetilde{G}$ in $L_{p}\left(\mathrm{R}^{k}\right)$. Define $\widetilde{E}: \tilde{\mathrm{g}} \times \mathrm{R}^{k} \rightarrow \mathrm{R}$ by

$$
\widetilde{E}(\tilde{a}, x)=E(\pi(\tilde{a}), x) .
$$

Let $\tilde{a}=\sum_{i=1}^{\tilde{d}} \tilde{\xi}_{i} \tilde{a}_{i} \in \tilde{\mathfrak{g}}$. Since $\pi\left(\tilde{a}_{i}\right) \in \pi([\tilde{\mathfrak{g}}, \tilde{\mathfrak{g}}])=[\mathfrak{g}, \mathfrak{g}] \subset \operatorname{span}\left\{a_{d^{\prime}+1}, \ldots, a_{d}\right\}$ for all $i \in\left\{d^{\prime}+1, \ldots, \tilde{d}\right\}$ and

$$
\pi(\tilde{a})=\sum_{i=1}^{d^{\prime}} \xi_{i} a_{i}+\sum_{i=d^{\prime}+1}^{\tilde{d}} \xi_{i} \pi\left(\tilde{a}_{i}\right)
$$

it follows that $\tilde{\xi}_{\tilde{a}}^{(0)}=\xi_{\pi(\tilde{a})}^{(0)}$, where

$$
\tilde{\xi}_{\tilde{a}}^{(0)}=\left(\tilde{\xi}_{1}, \ldots, \tilde{\xi}_{k}\right) .
$$

Hence

$$
(\widetilde{U}(\widetilde{\exp } \tilde{a}) \varphi)(x)=e^{i \widetilde{E}(\tilde{a}, x)} \varphi\left(x+\tilde{\xi}_{\tilde{a}}^{(0)}\right)
$$

for all $\varphi \in C_{c}\left(\mathrm{R}^{k}\right), \tilde{a} \in \tilde{\mathfrak{g}}$ and $x \in \mathrm{R}^{k}$, where $\widetilde{\exp }$ is the exponential map on $\tilde{\mathfrak{g}}$. Thus the representation $\widetilde{U}$ is of the same type as the representation $U$.

Note that $d \widetilde{U}\left(\tilde{a}_{i}\right)=d U\left(a_{i}\right)$ for all $i \in\left\{1, \ldots, d^{\prime}\right\}$. Therefore we can just as well use the group $\widetilde{G}$ with the representation $\widetilde{U}$ instead of the group $G$ with the representation $U$. According to Theorem 1.1 of [9] for all $t>0$ there exists a $\widetilde{K}_{t} \in \mathscr{S}(\widetilde{G})$ such that

$$
S_{t} \varphi=\int_{\widetilde{G}} d \tilde{g} \widetilde{K}_{t}(\tilde{g}) \widetilde{U}(\tilde{g}) \varphi
$$


for all $\varphi \in L_{p}\left(\mathrm{R}^{k}\right)$. Moreover, there exist $c, \tau>0$ and $\omega \in \mathrm{R}$ such that

$$
\left|\widetilde{K}_{t}(\widetilde{\exp }(\tilde{a}))\right| \leq c t^{-\widetilde{D} / m} e^{\omega t} e^{-\tau\left(|\tilde{a}|^{m} t^{-1}\right)^{1 /(m-1)}}
$$

for all $t>0$ and $\tilde{a} \in \tilde{\mathfrak{g}}$, where $\widetilde{D}=w_{1}+\ldots+w_{\tilde{d}}$ and the modulus $|\cdot|$ on $\tilde{\mathfrak{g}}$ is defined by

$$
\left|\sum_{i=1}^{\tilde{d}} \xi_{i} \tilde{a}_{i}\right|^{2 \tilde{w}}=\sum_{i=1}^{\tilde{d}}\left|\xi_{i}\right|^{2 \tilde{w} / w_{i}}
$$

and $\tilde{w}=\operatorname{lcm}\left(w_{1}, \ldots, w_{\tilde{d}}\right)$. By scaling one can take $\omega=0$ in case the operator $\widetilde{H}$ is homogeneous. Next, set $\tilde{\mathfrak{h}}=\operatorname{span}\left\{\tilde{a}_{k+1}, \ldots, \tilde{a}_{\tilde{d}}\right\}$ and for $y=$ $\left(y_{1}, \ldots, y_{k}\right) \in \mathrm{R}^{k}$ define $\hat{y} \in \tilde{\mathfrak{g}}$ by

$$
\hat{y}=y_{1} \tilde{a}_{1}+\ldots+y_{k} \tilde{a}_{k} .
$$

Since $\widetilde{E}$ is real valued one can define for all $t>0$ the function $\kappa_{t} \in C^{\infty}\left(\mathrm{R}^{k} \times \mathrm{R}^{k}\right)$ by

$$
\kappa_{t}(x ; y)=\int_{\tilde{\mathrm{h}}} d \tilde{b} \widetilde{K}_{t}(\widetilde{\exp }(\tilde{b}-\hat{x}+\hat{y})) e^{i \widetilde{E}(\tilde{b}-\hat{x}+\hat{y}, x)} .
$$

Then it is easy to verify that Statement II of Theorem 2.1 is valid.

Now we prove Statement III. It follows from the Gaussian bounds (3) that

$$
\begin{aligned}
\left|\kappa_{t}(x ; y)\right| \leq & \int_{\tilde{\mathrm{h}}} d \tilde{b} c t^{-\widetilde{D} / m} e^{\omega t} e^{-\tau\left(|\tilde{b}-\hat{x}+\hat{y}|^{m} t^{-1}\right)^{1 /(m-1)}} \\
\leq & c t^{-\widetilde{D} / m} e^{\omega t} \int_{\tilde{\mathrm{h}}} d \tilde{b} e^{-2^{-1} \tau\left(|\hat{x}-\hat{y}|^{m} t^{-1}\right)^{1 /(m-1)}} e^{-2^{-1} \tau\left(|\tilde{b}|^{m} t^{-1}\right)^{1 /(m-1)}} \\
= & c t^{-Q / m} e^{\omega t} e^{-2^{-1} \tau\left(|\hat{x}-\hat{y}|^{m} t^{-1}\right)^{1 /(m-1)}} \\
& \cdot\left(t^{-(\widetilde{D}-Q) / m} \int_{\tilde{\mathrm{h}}} d \tilde{b} e^{-2^{-1} \tau\left(|\tilde{b}|^{m} t^{-1}\right)^{1 /(m-1)}}\right)
\end{aligned}
$$

for all $t>0$ and $x, y \in \mathrm{R}^{k}$. But the quantity between the brackets is independent of $t$ (and also of $x$ and $y$ ), by scaling. Moreover, there exists a $\tau^{\prime}>0$ such that $\|z\| \leq \tau^{\prime}|\hat{z}|$ for all $z \in \mathrm{R}^{k}$ with $\|z\| \leq 1$. Hence, again by scaling, it follows that $\|z\| \leq \tau^{\prime}|\hat{z}|$ for all $z \in \mathrm{R}^{k}$. Therefore the proof of the Gaussian bounds of Statement III is complete if $\|\alpha\|=\|\beta\|=0$.

Next we consider derivatives of the reduced heat kernel $\kappa_{t}$. If $\alpha, \beta \in J\left(d^{\prime}\right)$ then

$$
\left(A^{\alpha} B^{\beta} \kappa_{t}\right)(x ; y)=\int_{\tilde{h}} d \tilde{b}\left(\widetilde{A}^{\alpha} \widetilde{B}^{\beta} \widetilde{K}_{t}\right)(\widetilde{\exp }(\tilde{b}-\hat{x}+\hat{y})) e^{i \widetilde{E}(\tilde{b}-\hat{x}+\hat{y}, x)}
$$


for all $t>0$ and $x, y \in \mathrm{R}^{k}$, where $\widetilde{B}_{i}=d R_{\widetilde{G}}\left(\tilde{a}_{i}\right)$ for all $i \in\left\{1, \ldots, d^{\prime}\right\}$ and $R_{\widetilde{G}}$ is the right regular representation on $\widetilde{G}$. Since one has Gaussian bounds

$$
\left|\left(\widetilde{A}^{\alpha} \widetilde{B}^{\beta} \widetilde{K}_{t}\right)(\widetilde{\exp }(\tilde{a}))\right| \leq c t^{-(\widetilde{D}+\|\alpha\|+\|\beta\|) / m} e^{\omega t} e^{-\tau\left(|\tilde{a}|^{m} t^{-1}\right)^{1 /(m-1)}}
$$

by [9], Theorem 1.1, one can estimate $A^{\alpha} B^{\beta} \kappa_{t}$ as in (4). Again $\omega$ can be taken equal to 0 if $\widetilde{H}$ is homogeneous.

Finally, since $A_{i}=\partial / \partial x_{i}+M_{i}$ for all $i \in\{1, \ldots k\}$ with $M_{i}$ a multiplication operator with a polynomial it follows from the Gaussian bounds that $S_{t}$ maps $\mathscr{S}\left(\mathrm{R}^{k}\right)$ into $\mathscr{S}\left(\mathrm{R}^{k}\right)$ for all $t>0$. Therefore $\mathscr{S}\left(\mathrm{R}^{k}\right)$ is a core for $\bar{H}$ (see [2] Corollary 3.1.7). This completes the proof of Theorem 2.1.

\section{Applications and examples}

The Gaussian bounds have several implications. The first is the $p$-independence of the spectrum if all weights equal one. Note that it will follow from Theorem 4.2 that the operator $H$ is already closed on $L_{p}$ for all $p \in\langle 1, \infty\rangle$.

COROLlary 3.1. Assume the notation and conditions of Theorem 2.1. Moreover, suppose that $w_{i}=1$ for all $i \in\left\{1, \ldots, d^{\prime}\right\}$. Then for all $p \in[1, \infty]$ the spectrum $\sigma_{p}(\bar{H})$ of the operator $\bar{H}$ on $L_{p}\left(\mathrm{R}^{k}\right)$ is independent of $p$.

ProOF. This follows from [15], or [16].

Note that the spectrum $\sigma(\bar{H})$ is independent of $p \in[1, \infty]$ if the representation $U$ is irreducible, by the arguments given in the proof of Theorem 2.5 of [8].

The second implication of the Gaussian bounds is that the bounded $H_{\infty}$ functional calculus on $L_{2}$ extends to all $L_{p}$ spaces.

Corollary 3.2. Assume the notation and conditions of Theorem 2.1. Then for all $p \in\langle 1, \infty\rangle$ and large enough $\lambda>0$ the operator $\bar{H}+\lambda I$ has a bounded $H_{\infty}$-functional calculus on $L_{p}\left(\mathrm{R}^{k}\right)$. If the operator $\bar{H}$ is homogeneous then one can take $\lambda=0$.

Proof. By [9] Theorem 9.2.III the semigroup generator $\bar{H}$ satisfies a Gårding inequality on $L_{2}$. Therefore $\bar{H}+\lambda I$ is maximal accretive if $\lambda>0$ is large enough. Hence it follows from Theorem $\mathrm{G}$ of [1] that the operator $\bar{H}+\lambda I$ has a bounded $H_{\infty}$-functional calculus on $L_{2}$. Then the corollary is a consequence of the Gaussian bounds of Theorem 2.1.III and [6] Theorem 3.4.

Quadrature of the Gaussian bounds gives semigroup bounds.

Corollary 3.3. Assume the notation and conditions of Theorem 2.1. Then there exist $c>0$ and $\omega \in \mathrm{R}$ such that for all $p, q \in[1, \infty]$ with $p \leq q$ one has

$$
\left\|S_{t}\right\|_{p \rightarrow q} \leq c t^{-Q(1 / p-1 / q) / m} e^{\omega t} \quad \text { uniformly for all } t>0 .
$$


Proof. Obviously $\left\|S_{t}\right\|_{1 \rightarrow \infty}=\left\|\kappa_{t}\right\|_{\infty} \leq c t^{-Q / m} e^{\omega t}$ by the bounds of Theorem 2.1.III. Next, let $c, \tau$ be as in Theorem 2.1.III with $\|\alpha\|=\|\beta\|=0$. For $t>0$ define $G_{t}: \mathrm{R}^{k} \rightarrow \mathrm{R}$ by $G_{t}(x)=c t^{-Q / m} e^{-\tau\left(\|x-y\|^{m} t^{-1}\right)^{1 /(m-1)}}$. Then

$$
\left|\left(S_{t} \varphi\right)(x)\right| \leq e^{\omega t} \int_{\mathrm{R}^{k}} d y G_{t}(x-y)|\varphi(y)|=e^{\omega t}\left(G_{t} *|\varphi|\right)(x)
$$

for all $\varphi \in C_{c}^{\infty}\left(\mathrm{R}^{k}\right)$ and $x \in \mathrm{R}^{k}$, where $*$ denotes the convolution on the commutative group $\mathrm{R}^{k}$. Therefore, $\left\|S_{t}\right\|_{p \rightarrow p} \leq\left\|G_{t}\right\|_{1} e^{\omega t}=\left\|G_{1}\right\|_{1} e^{\omega t}$ for all $t>0$ and $p \in[1, \infty]$, by scaling. Now the corollary follows by interpolation.

If the spectrum $\sigma(\bar{H})$ of $\bar{H}$ is a subset of $\langle 0, \infty\rangle$ then one also has exponential decay for $t \rightarrow \infty$ in the Gaussian bounds. Furthermore, the decay at infinity of the kernel is almost equal to the growth bound of the semigroup on $L_{2}$.

Proposition 3.4. Assume the notation and conditions of Theorem 2.1. Let

$$
\lambda_{1}=\inf \left\{\operatorname{Re}(\varphi, H \varphi): \varphi \in C_{c}^{\infty}\left(\mathrm{R}^{k}\right)\right\} .
$$

Then for all $\varepsilon>0$ there exist $c, \tau>0$ such that

$$
\left|\kappa_{t}(x ; y)\right| \leq c t^{-Q / m} e^{-\left(\lambda_{1}-\varepsilon\right) t} e^{-\tau\left(\|x-y\|^{m} t^{-1}\right)^{1 /(m-1)}}
$$

for all $t>0$ and $x, y \in \mathrm{R}^{k}$.

Proof. It follows from semigroup theory that $\left\|S_{t}\right\|_{2 \rightarrow 2} \leq e^{-\lambda_{1} t}$ for all $t>0$. Let $\varepsilon>0$. Then by Corollary 3.3 there exist $c, \omega>0$ such that

$$
\begin{aligned}
\left\|\kappa_{t}\right\|_{\infty} & =\left\|S_{t}\right\|_{1 \rightarrow \infty} \leq\left\|S_{\varepsilon t / 2}\right\|_{1 \rightarrow 2}\left\|S_{(1-\varepsilon) t}\right\|_{2 \rightarrow 2}\left\|S_{\varepsilon t / 2}\right\|_{2 \rightarrow \infty} \\
& \leq c t^{-Q / m} e^{-\lambda_{1}(1-\varepsilon) t} e^{\varepsilon \omega t}
\end{aligned}
$$

for all $t>0$. Hence interpolation with the Gaussian estimates of Theorem 2.1.III gives

$$
\begin{aligned}
\left|\kappa_{t}(x ; y)\right| & =\left|\kappa_{t}(x ; y)\right|^{\varepsilon}\left|\kappa_{t}(x ; y)\right|^{1-\varepsilon} \\
& \leq\left(c t^{-Q / m} e^{\omega t} e^{-\tau\left(\|x-y\|^{m} t^{-1}\right)^{1 /(m-1)}}\right)^{\varepsilon}\left(c t^{-Q / m} e^{-\lambda_{1}(1-\varepsilon) t} e^{\varepsilon \omega t}\right)^{1-\varepsilon} \\
& =c t^{-Q / m} e^{-\lambda_{1}(1-\varepsilon)^{2} t} e^{\varepsilon(2-\varepsilon) \omega t} e^{-\tau \varepsilon\left(\|x-y\|^{m} t^{-1}\right)^{1 /(m-1)}}
\end{aligned}
$$

for all $t>0$ and $x, y \in \mathrm{R}^{k}$, from which the proposition follows.

Example 3.5. Let $j, n \in \mathrm{N}$. Then the anharmonic oscillator is the operator

$$
H_{0}=\left(-\frac{d^{2}}{d x^{2}}\right)^{j}+x^{2 n}
$$


on $L_{2}(\mathrm{R})$ and domain the Schwartz space. This operator is a special example for which Theorem 1.1 applies in the following way. Let $G$ be the connected simply connected Heisenberg group with Lie algebra $\mathrm{g}$ and let $a_{1}, a_{2}, a_{3}$ be a basis of $g$ such that $\left[a_{1}, a_{2}\right]=a_{3}$. Then the standard irreducible representation $U$ of $G$ is given by

$$
\left(U\left(\exp \left(\xi_{1} a_{1}+\xi_{2} a_{2}+\xi_{3} a_{3}\right)\right) \varphi\right)(x)=e^{i \xi_{3}} e^{i x \xi_{2}} \varphi\left(x+\xi_{1}\right)
$$

for all $\varphi \in C_{c}^{\infty}(\mathrm{R})$. So $U$ is of the form (1) and if one takes $k=1$ and $d^{\prime}=2$. Then $A_{1}=-i P$ and $A_{2}=i Q$, where $P$ and $Q$ are the self-adjoint operators in $L_{2}(\mathrm{R})$ given by $(P f)(x)=i f^{\prime}(x)$ and $(Q f)(x)=x f(x)$ for all $f \in C_{c}^{\infty}(\mathrm{R})$ and $x \in \mathrm{R}$. If

$$
H=(-1)^{j} A_{1}^{2 j}+(-1)^{n} A_{2}^{2 n}
$$

then the operator $H_{0}$ is the restriction of the self-adjoint operator $H$ to the Schwartz space, which is a core for $H$ (see [7], Example 7.1). Let $d_{0}=$ $\operatorname{gcd}(j, n)$. Then $w_{1}=n / d_{0}, w_{2}=j / d_{0}, Q=n / d_{0}$ and the weighted order of $H$ equals $m=2 j n / d_{0}$. Moreover, $\|x\|=|x|$ for all $x \in \mathrm{R}$.

Let $\kappa$ be the reduced heat kernel of the semigroup generated by $H$. Then it is a consequence of Theorem 1.1 that there are $c, \tau>0$ such that

$$
\left|\kappa_{t}(x ; y)\right| \leq c t^{-1 /(2 j)} e^{-\tau\left(|x-y|^{2 j n / d_{0}} t^{-1}\right)^{1 /\left(2 j n / d_{0}-1\right)}}
$$

for all $t>0$ and $x, y \in \mathrm{R}$. Moreover, if $j=n=1$ then the smallest eigenvalue of $H$ equals $\lambda_{1}=1$ and it follows from Proposition 3.4 that for all $\varepsilon>0$ there are $c, \tau>0$ such that

$$
\left|\kappa_{t}(x ; y)\right| \leq c t^{-1 / 2} e^{-(1-\varepsilon) t} e^{-\tau|x-y|^{2} t^{-1}}
$$

for all $t>0$ and $x, y \in \mathrm{R}$. Note that these bounds are consistent with the explicit expression for $\kappa$ by Mehler's formula;

$$
\kappa_{t}(x ; y)=\left(\pi\left(1-e^{-4 t}\right)\right)^{-1 / 2} e^{-(x+y)^{2}(\tanh t) / 4} e^{-(x-y)^{2}(\operatorname{coth} t) / 4} e^{-t}
$$

for all $t>0$ and $x, y \in \mathrm{R}$ (see [3] Theorem 7.13).

Lower order terms are also allowed. If, for example, $H$ is an operator of the form

$$
H=\left(-\frac{d^{2}}{d x^{2}}\right)^{j}+\mu x^{2 n}+\sum_{\substack{l, k \geq 0 \\ l j+k n<2 j n}} c_{k l} x^{l} \frac{d^{k}}{d x^{k}}
$$

with $\mu>0, c_{k l} \in \mathbf{C}$ and domain $D(H)=D\left(P^{2 j}\right) \cap D\left(Q^{2 n}\right)$ then the semigroup generated by $H$ has a smooth reduced heat kernel $\kappa$. Moreover, 
there are $c, \tau, \omega>0$ such that

$$
\left|\kappa_{t}(x ; y)\right| \leq c t^{-1 /(2 j)} e^{\omega t} e^{-\tau\left(|x-y|^{2 j n / d_{0}} t^{-1}\right)^{1 /\left(2 j n / d_{0}-1\right)}}
$$

for all $t>0$ and $x, y \in \mathrm{R}$. A typical example of an operator $H$ in (5) is the operator

$$
H=-\frac{d^{2}}{d x^{2}}+\mu x^{4}+\mu^{\prime} x^{2}
$$

with $\mu>0$ and $\mu^{\prime} \in \mathrm{R}$.

EXAMPLE 3.6. Let $\mathfrak{m}$ be an ideal in the Lie algebra $\mathfrak{g}$ of a connected simply connected Lie group $G$, let $M$ be the connected (and simply connected) subgroup of $G$ with Lie algebra $m$ and let $\chi$ be a one dimensional representation of $M$. Set $k=d-\operatorname{dim} \mathfrak{m}$. Let $a_{1}, \ldots, a_{d}$ be a basis for $g$ such that $a_{k+1}, \ldots, a_{d}$ is a basis for $\mathfrak{m}$. Finally, assume that $d^{\prime} \geq k$ is such that $a_{1}, \ldots, a_{d^{\prime}}$ is an algebraic basis for $\mathfrak{g}$ and $[\mathfrak{g}, \mathfrak{g}] \subset\left\{a_{d^{\prime}+1}, \ldots, a_{d}\right\}$. Then the basis realization of the induced representation $\operatorname{Ind}(M \uparrow G, \chi)$ is of the form (1). This follows immediately from the description in [5] p. 125 and the fact that $\mathfrak{m}$ is an ideal.

As an example reconsider the Hamiltonian with polynomial vector field $\vec{A}$ and magnetic field $\vec{B}=\vec{\nabla} \times \vec{A}$. In order to avoid confusion between the components of the vector field $\vec{A}$ and the infinitesimal generators $A_{j}$ which we introduce below, we denote the components of the components of the vector field $\vec{A}$ by $A_{j}^{(M)}$. Set $X_{j}=\hbar \partial_{j}-i e c^{-1} A_{j}^{(M)}$ with domain $\mathscr{S}\left(\mathrm{R}^{3}\right)$ for all $j \in\{1,2,3\}$. Then $\left[X_{i}, X_{j}\right]=-i \hbar e c^{-1} \sum_{k=1}^{3} \varepsilon_{i j k} B_{k}$ for all $i, j \in\{1,2,3\}$. Since multiplication operators commute and the $B_{j}$ are polynomials it follows that $X_{1}, X_{2}, X_{3}$ generate a finite dimensional Lie subalgebra $g$ of operators in $\operatorname{Hom}\left(\mathscr{S}\left(\mathrm{R}^{3}\right)\right)$, the space of all linear operators acting on the Schwartz space $\mathscr{S}\left(\mathrm{R}^{3}\right)$. Extend $X_{1}, X_{2}, X_{3}$ to a basis $X_{1}, X_{2}, X_{3}, \ldots, X_{d}$ for $g$ such that $X_{4}, \ldots, X_{d}$ are all polynomial multiplication operators, say with polynomials $\psi_{4}, \ldots, \psi_{d}$. Then $\mathfrak{m}=\operatorname{span}\left\{X_{4}, \ldots, X_{d}\right\}$ is an (Abelian) ideal in $\mathrm{g}$. Moreover, there exists a unique linear map $l: \mathfrak{g} \rightarrow \mathbf{C}$ such that $l\left(X_{j}\right)=-e c^{-1} A_{j}^{(M)}(0)$ for all $j \in\{1,2,3\}$ and $l\left(X_{j}\right)=-i \psi_{j}(0)$ for all $j \in\{4, \ldots, d\}$. Define $\chi: M \rightarrow \mathbf{C}$ by $\chi(\exp a)=\exp (i l(a))$ for all $a \in \mathfrak{m}$. Then it follows from [12] Proposition II.1.6.1 and its proof that $\chi$ is a one dimensional representation of $M$ and that the basis realization $U$ of the induced representation Ind $(M \uparrow G, \chi)$ with respect to the basis $a_{1}, \ldots, a_{d}$ given by $a_{j}=\hbar^{-1} X_{j}$ is of the form (1). For $j \in\{1,2,3\}$ let $A_{j}=d U\left(a_{j}\right)$ be the associated infinitesimal generator. Note that $X_{j} \varphi=\hbar A_{j} \varphi$ for all $\varphi \in \mathscr{S}\left(\mathrm{R}^{3}\right)$. One can take $d^{\prime}=3$ and set $H=-\frac{\hbar^{2}}{2 m}\left(A_{1}^{2}+A_{2}^{2}+A_{3}^{2}\right)$. By Theorem 2.1.IV it follows that $\bar{H}$ is the 
closure of the operator

$$
H_{0}=\frac{1}{2 m}\left(\vec{p}-\frac{e}{c} \vec{A}\right)^{2}=-\frac{1}{2 m}\left(X_{1}^{2}+X_{2}^{2}+X_{3}^{2}\right)
$$

with domain $\mathscr{S}\left(\mathrm{R}^{3}\right)$. Moreover, Theorem 2.1 states that the semigroup generated by $\bar{H}$ has a reduced heat kernel $\kappa$ and there are $c, \tau>0$ such that

$$
\left|\kappa_{t}(x ; y)\right| \leq c t^{-3 / 2} e^{-\tau|x-y|^{2} t^{-1}}
$$

for all $t>0$ and $x, y \in \mathrm{R}^{3}$, where $|x-y|$ is the Euclidean modulus of $x-y$.

REMARK 3.7. It can be proved as above that any operator associated with a representation of the form (1) equals an operator associated with an induced representation as described in the first part of Example 3.6 on a possibly different Lie group.

\section{Riesz transforms}

If $H=\sum_{\|\alpha\|=m} c_{\alpha} A^{\alpha}$ is acting on $L_{p}\left(\mathrm{R}^{k}\right)$ and is such that the comparable operator $\widetilde{H}=\sum_{\|\alpha\|=m} c_{\alpha} \widetilde{A}^{\alpha}$ is a homogeneous weighted subcoercive operator then in this section we show that the Riesz transforms of all orders are bounded on $L_{p}\left(\mathrm{R}^{k}\right)$ for all $p \in\langle 1, \infty\rangle$. The result relies on an application of the transference theorem in [4], which holds naturally for kernels in $L_{1}(\widetilde{G})$, and a technique that has been used in the the study of the Riesz transforms of all orders for homogeneous subcoercive operator with complex coefficients in [10] Section 4. We stress that in the present context the representation of the nilpotent group $G$ can be any representation induced from a character, including the basis realization of a unitary irreducible representation (see [14], [5] and [8], Lemma 2.1).

TheOREM 4.1. Let $(\mathscr{M}, \mu)$ be a $\sigma$-finite measure space, $p \in\langle 1, \infty\rangle$ and $U$ a continuous bounded representation of $G$ in $L_{p}(\mathscr{M})$. Suppose $\widetilde{H}=$ $\sum_{\|\alpha\|=m} c_{\alpha} \widetilde{A}^{\alpha}$ is a homogeneous weighted subcoercive operator of order $m$ on $\widetilde{G}$ and set

$$
H=\sum_{\|\alpha\|=m} c_{\alpha} A^{\alpha}
$$

with domain $D(H)=\bigcap_{\|\alpha\|=m} D\left(A^{\alpha}\right)$. Then $H$ is closed, generates a bounded semigroup and for all $\alpha \in J\left(d^{\prime}\right)$ one has $D\left(H^{\|\alpha\| / m}\right) \subset D\left(A^{\alpha}\right)$. Moreover, there exists a $c>0$ such that

$$
\left\|A^{\alpha} \varphi\right\|_{p} \leq c\left\|H^{\|\alpha\| / m} \varphi\right\|_{p}
$$


for all $\varphi \in D\left(H^{\|\alpha\| / m}\right)$.

Proof. Let $\Phi: \widetilde{G} \rightarrow G$ be as in the proof of Theorem 2.1. Let $\widetilde{U}=U \circ \Phi$. Then $\widetilde{U}$ is a continuous representation of $\widetilde{G}$ in $L_{p}(\mathscr{M})$. If $\widetilde{K}$ is the kernel of the semigroup $\widetilde{S}$ generated by $\widetilde{H}$ and $S$ the semigroup generated by $\bar{H}$ then $S_{t} \varphi=\int_{\widetilde{G}} d \tilde{g} \widetilde{K}(\tilde{g}) \widetilde{U}(\tilde{g}) \varphi$. for all $t>0$ and $\varphi \in L_{p}(\mathscr{M})$. Then the transference method of [4], Theorem 2.4, together with a density argument, gives the bounds

$$
\left\|S_{t}\right\|_{p \rightarrow p} \leq c^{2}\left\|\widetilde{S}_{t}\right\|_{\tilde{p} \rightarrow \tilde{p}} \leq c^{2}\left\|\widetilde{K}_{t}\right\|_{\tilde{1}}=c^{2}\left\|\widetilde{K}_{1}\right\|_{\tilde{1}}
$$

uniformly for all $t>0$, where $\|\cdot\|_{\tilde{p}}$ is the norm on $L_{p}(\widetilde{G})$ and we have set $c=\sup _{g \in G}\|U(g)\|_{p \rightarrow p}$. So $\bar{H}$ generates a bounded semigroup.

If $n \in \mathrm{N}$ is large enough then for all $v, \varepsilon>0$ the convolution kernel $\tilde{k}_{\alpha ; \nu, \varepsilon}$ of the operator

$$
\widetilde{R}_{\alpha ; \nu, \varepsilon}=\widetilde{A}^{\alpha}(\nu I+\widetilde{H})^{-\|\alpha\| / m}(I+\varepsilon \widetilde{H})^{-n}
$$

is in $L_{1}(\widetilde{G})$. Since $A_{i}=d U\left(a_{i}\right)=d \widetilde{U}\left(\tilde{a}_{i}\right)$ for all $i \in\left\{1, \ldots, d^{\prime}\right\}$ it follows that

$$
R_{\alpha ; v, \varepsilon}=A^{\alpha}(v I+\bar{H})^{-\|\alpha\| / m}(I+\varepsilon \bar{H})^{-n}=\int_{\widetilde{G}} d \tilde{g} \tilde{k}_{\alpha ; v, \varepsilon}(\tilde{g}) \widetilde{U}(\tilde{g}) .
$$

The transference method then gives the estimates

$$
\left\|R_{\alpha ; \nu, \varepsilon}\right\|_{p \rightarrow p} \leq c^{2}\left\|\widetilde{R}_{\alpha ; \nu, \varepsilon}\right\|_{\tilde{p} \rightarrow \tilde{p}}
$$

uniformly for all $\nu, \varepsilon>0$. But the right hand side of (6) is bounded uniformly for all $\nu, \varepsilon>0$ by scaling on $\widetilde{G}$ (cf. [10] Lemma 4.1). Hence there exists an $M>0$ such that $\left\|R_{\alpha ; \nu, \varepsilon}\right\|_{p \rightarrow p} \leq M$ uniformly for all $v, \varepsilon>0$. Then

$$
\left\|A^{\alpha} \varphi\right\|_{p} \leq M\left\|(v I+\bar{H})^{\|\alpha\| / m}(I+\varepsilon \bar{H})^{n} \varphi\right\|_{p}
$$

for all $\varphi \in D_{\infty}(\bar{H})=\bigcap_{\beta \in J\left(d^{\prime}\right)} D\left(A^{\beta}\right)$. Taking the limit $\varepsilon \downarrow 0$ it follows that

$$
\left\|A^{\alpha} \varphi\right\|_{p} \leq M\left\|(v I+\bar{H})^{\|\alpha\| / m} \varphi\right\|_{p}
$$

for all $\varphi \in D_{\infty}(\bar{H})$.

Now let $v>0, N \in \mathrm{N}$ and $\varphi \in D\left(\bar{H}^{N / m}\right)=D\left((v I+\bar{H})^{N / m}\right)$. Since $D_{\infty}(\bar{H})$ is a core for the operator $(\nu I+\bar{H})^{N / m}$ there are $\varphi_{1}, \varphi_{2}, \ldots \in D_{\infty}(\bar{H})$ such that $\lim \varphi_{n}=\varphi$ and $\lim (\nu I+\bar{H})^{N / m} \varphi_{n}=(\nu I+\bar{H})^{N / m} \varphi$. Then $\lim (\nu I+$ $\bar{H})^{j / m} \varphi_{n}=(\nu I+\bar{H})^{j / m} \varphi$ for all $j \in\{0,1, \ldots, N\}$. Hence by induction on the number of indices of the multi-index $\alpha$ and the closedness of the $A_{i}$ it 
follows from the estimates (7) that $\lim A^{\alpha} \varphi_{n}=A^{\alpha} \varphi$ for all $\alpha$ with $\|\alpha\| \leq N$. So $D\left(\bar{H}^{\|\alpha\| / m}\right) \subset D\left(A^{\alpha}\right)$ for all $\alpha \in J\left(d^{\prime}\right)$ and the estimates (7) are valid uniformly for all $\varphi \in D\left(\bar{H}^{\|\alpha\| / m}\right)$ and $v>0$. Taking the limit $v \downarrow 0$ yields $\left\|A^{\alpha} \varphi\right\|_{p} \leq M\left\|\bar{H}^{\|\alpha\| / m} \varphi\right\|_{p}$ for all $\varphi \in D\left(\bar{H}^{\|\alpha\| / m}\right)$.

Finally, one has as a special case that $D(\bar{H}) \subset D\left(A^{\alpha}\right)$ for all $\alpha$ with $\|\alpha\|=m$. Therefore the operator $H$ is closed.

In the unweighted case, i.e., if $w_{1}=\ldots=w_{d^{\prime}}=1$, then one can prove as in Corollary 4.3 of [10] that

$$
D\left(H^{n w / m}\right)=\bigcap_{\|\alpha\|=n w} D\left(A^{\alpha}\right)
$$

for all $n \in \mathrm{N}$ and that the seminorms on the two spaces are equivalent. It is unclear whether the equality (8) is also valid in the weighted case.

Finally, for non-homogeneous operators we prove optimal regularity for any weighted subcoercive operator.

Theorem 4.2. Let $(\mathscr{M}, \mu)$ be a $\sigma$-finite measure space, $p \in\langle 1, \infty\rangle$ and $U$ a continuous bounded representation of $G$ in $L_{p}(\mathscr{M})$. Suppose $\widehat{H}=$ $\sum_{\|\alpha\| \leq m} c_{\alpha} \widehat{A}^{\alpha}$ is a weighted subcoercive operator of order $m$ on $G$, where $L_{G}$ is the left regular representation on $L_{p}(G)$ and $\widehat{A}_{i}=d L_{G}\left(a_{i}\right)$ for all $i \in\left\{1, \ldots, d^{\prime}\right\}$. Let

$$
H=\sum_{\|\alpha\| \leq m} c_{\alpha} A^{\alpha}
$$

be the corresponding operator on $L_{p}(\mathscr{M})$. Then $H$ is closed and for all $\alpha \in$ $J\left(d^{\prime}\right)$ one has $D\left((H+\lambda I)^{\|\alpha\| / m}\right) \subset D\left(A^{\alpha}\right)$ if $\lambda>0$ is large enough. Moreover, there exists a $c>0$ such that

$$
\left\|A^{\alpha} \varphi\right\|_{p} \leq c\left\|(H+\lambda I)^{\|\alpha\| / m} \varphi\right\|_{p}
$$

for all $\varphi \in D\left((H+\lambda I)^{\|\alpha\| / m}\right)$.

Proof. We may assume that $\widehat{H}$ generates an exponentially decreasing semigroup $\widehat{S}$ on $L_{p}(G)$. The proof of the theorem is similar to the proof of Theorem 4.1. If $n \in \mathrm{N}$ is large enough then for all $\varepsilon>0$ the convolution kernel of the operator

$$
\widehat{R}_{\alpha ; \varepsilon}=\widehat{A}^{\alpha} \widehat{H}^{-\|\alpha\| / m}(I+\varepsilon \widehat{H})^{-n}
$$

is in $L_{1}(G)$. Moreover, $D\left(\widehat{H}^{\|\alpha\| / m}\right) \subset D\left(\widehat{A}^{\alpha}\right)$ and the embedding is continuous in $L_{p}(G)$-sense by [9], Section 9. Next,

$$
\left\|\widehat{R}_{\alpha ; \varepsilon}\right\|_{L_{p}(G) \rightarrow L_{p}(G)} \leq\left\|\widehat{A}^{\alpha} \widehat{H}^{-\|\alpha\| / m}\right\|_{L_{p}(G) \rightarrow L_{p}(G)}\left\|(I+\varepsilon \widehat{H})^{-n}\right\|_{L_{p}(G) \rightarrow L_{p}(G)}
$$


and if $M=\sup _{t>0}\left\|\widehat{S}_{t}\right\|_{L_{p}(G) \rightarrow L_{p}(G)}$ then

$\left\|(I+\varepsilon \widehat{H})^{-n}\right\|_{L_{p}(G) \rightarrow L_{p}(G)} \leq(n-1) !^{-1} \int_{0}^{\infty} d t e^{-t} t^{n-1}\left\|\widehat{S}_{\varepsilon t}\right\|_{L_{p}(G) \rightarrow L_{p}(G)} \leq M$

uniformly for $\varepsilon>0$. Therefore the operators $\widehat{R}_{\alpha ; \varepsilon}$ are bounded on $L_{p}(G)$ uniformly for $\varepsilon>0$. The rest of the proof is by the same arguments as in the proof of Theorem 4.1. It relies on the transference method.

Note that in fact the above argument can be applied to any continuous bounded representation of an amenable Lie group $G$ in $L_{p}(\mathscr{M})$ where $(\mathscr{M}, \mu)$ is a $\sigma$-finite measure space.

ACKNOWLEDGEMENTS. This work was completed while the second named author was visiting the Eindhoven University of Technology. He wishes to thank for the hospitality. This research was partially supported by FONDECYT 1970722 and DICYT 049633PC.

Both authors wish to thank Derek Robinson for his suggestions and comments.

\section{REFERENCES}

1. Albrecht, D., Duong, X. and McIntosh, A., Operator theory and harmonic analysis, in Instructional Workshop on Analysis and Geometry, Part III, vol. 34 of Proceedings of the Centre for Mathematics and its Applications. Australian National University, Canberra, 1996, 77-136.

2. Bratteli, O. and Robinson, D. W., Operator Algebras and Quantum Statistical Mechanics, vol. 1. Second edition. Springer-Verlag, New York etc., 1987.

3. Davies, E. B., One-parameter Semigroups, London Math. Soc. Monographs 15 (1980).

4. Coifman, R. R., and Weiss, G., Transference Methods in Analysis, CBMS Regional Conference Series in Mathematics 31. Amer. Math. Soc., Providence, 1977.

5. Corwin, L. and Greenleaf, F. P., Representations of Nilpotent Lie Groups and their Applications Part 1: Basic theory and examples, Cambridge Stud. Adv. Math. 18 (1990).

6. Duong, X. T. and Robinson, D. W., Semigroup Kernels, Poisson Bounds, and Holomorphic Functional Calculus, J. Funct. Anal. 142 (1996), 89-129.

7. Elst, A. F. M. ter and Robinson, D. W., Weighted strongly elliptic operators on Lie groups, J. Funct. Anal. 125 (1994), 548-603.

8. Elst, A. F. M. ter and Robinson, D. W., Reduced heat kernels on nilpotent Lie groups, Comm. Math. Phys. 173 (1995), 475-511.

9. Elst, A. F. M. ter and Robinson, D. W., Weighted subcoercive operators on Lie groups, J. Funct. Anal. 157 (1998), 88-163.

10. Elst, A. F. M. ter, Robinson, D. W. and Sikora, A., Heat kernels and Riesz transforms on nilpotent Lie groups, Coll. Math. 74 (1997), 191-218.

11. Elst, A. F. M. ter and Smulders, C. M. P. A., Reduced heat kernels on homogeneous spaces, J. Operator Theory 42 (1999), 269-304.

12. Helffer, B. and Nourrigat, J., Hypoellipticité maximale pour des opérateurs polynomes de champs de vecteurs, Progress in Mathematics 58, Birkhäuser, Boston etc., 1985. 
13. Jørgensen, P. E. T. and Klink, W., Quantum mechanics and nilpotent groups I. The curved magnetic field, Publ. Res. Inst. Math. Sci. 21 (1985), 969-999.

14. Kirillov, A. A., Unitary representations of nilpotent Lie groups, Russian Math. Surveys 17 (1962), 53-104.

15. Kunstmann, P. C., Heat kernel estimates and $L^{p}$-spectral independence of elliptic operators, Bull. London Math. Soc. 31 (1999), 345-353.

16. Liskevich, V. and Vogt, H., On $L^{p}$-spectra and essential spectra of second order elliptic operators, Proc. London Math. Soc. 80 (2000), 590-610.

17. Nagel, A., Ricci, F. and Stein, E. M., Harmonic analysis and fundamental solutions on nilpotent Lie groups, in Sadosky, C., ed., Analysis and partial differential equations, Lecture Notes in Pure and Appl. Math. 122 (1990), 249-275.

18. Sikora, A., On-diagonal estimates on Schrödinger semigroup kernels and reduced heat kernels, Comm. Math. Phys. 188 (1997), 233-249.

19. Simon, B., Schrödinger semigroups, Bull. Amer. Math. Soc. 7 (1982), 447-526.

DEPARTMENT OF MATHEMATICS AND COMPUTING SCIENCE EINDHOVEN UNIVERSITY OF TECHNOLOGY

P.O. BOX 513

5600 MB EINDHOVEN

THE NETHERLANDS
DEPARTAMENTO DE MATEMÁTICA Y C.C. UNIVERSIDAD DE SANTIAGO DE CHILE CASILLA 307, CORREO 2, SANTIAGO CHILE 